\title{
EFEITO DA RADIAÇÃO GAMA E DOARMAZENAMENTO NA QUALIDADE DE PEDÚNCULOS DE CAJU (Anacardium occidentale L.)
}

\author{
Effect of gamma radiation and storage on cashew apple (Anacardium occidentale L.) quality \\ Adriana Régia Marques de Souza ${ }^{1}$, Solange Guidolin Canniatti Brazaca², Valter Arthur³, Anderson Giovani \\ Cândico Oliveira ${ }^{4}$, Marta Helena Fillet Spoto ${ }^{5}$, Júlio Marcos Melges Walder ${ }^{6}$
}

\begin{abstract}
RESUMO
Devido a altas perdas de pedúnculos de caju, métodos de conservação são necessários para um maior aproveitamento dos mesmos. Com esse objetivo, pedúnculos de caju vermelho, maduros, obtidos de cajueiro anão precoce, foram irradiados com doses de $0,0,5$ e $1,0 \mathrm{kGy}$ e armazenados por 9 dias, refrigerados $4{ }^{\circ} \mathrm{C}$ para verificação da estabilidade da qualidade durante o período de armazenamento. Para isso foram realizadas análises químicas, verificando mudanças nos teores de açúcares redutores, Brix, vitamina $\mathrm{C} \mathrm{e} \mathrm{pH}$. Alterações na firmeza e cor também foram observadas. Os teores de vitamina $\mathrm{C}$ diminuíram tanto em função do tempo de estocagem como em função das doses de radiação empregadas. A firmeza dos frutos foi influenciada tanto pelas diferentes doses de radiação quanto pelo armazenamento, aumentando durante o armazenamento e diminuindo quanto maiores as doses de irradiação. $\mathrm{O}$ método de conservação por irradiação mostrou-se eficiente para o prolongamento da vida de prateleira dos pedúnculos de caju e manutenção de sua qualidade pós-colheita.
\end{abstract}

Termos para indexação: Radiação, vitamina C, caju, armazenamento, cor.

\begin{abstract}
The non-efficiency or absence of techniques for adequate handling, transport, and storage of cashew stalks associated with a high level of perishability, generates high economic loss in the commercialization of these fruits, hence the development of conservation methods becomes a necessity in order to enhance the profitability. Cashew apples from precocious dwarf cashew tree were irradiated with doses of $0,0.5$, and $1.0 \mathrm{kGy}$ and stored during nine days under refrigeration at $4{ }^{\circ} \mathrm{C}$ for evaluation of the quality stability during storage. Chemical analyses were performed to verify changes in reducing sugars, Brix, vitamin $\mathrm{C}$, and $\mathrm{pH}$. Alterations in the firmness and color were also observed. The levels of vitamin $\mathrm{C}$ decreased as a function of storage as well as a function of the radiation doses employed. The firmness of the fruits was influenced by both the radiation doses and storage time, increasing during storage and decreasing as the radiation doses increased. Irradiation was shown to be efficient in the prolongation of the shelf-life of cashew stalks.
\end{abstract}

Index terms: Radiation, vitamin C, cashew, storage, color.

(Recebido em 9 de março de 2006 e aprovado em 30 de abril de 2008)

\section{INTRODUÇÃO}

Um dos frutos tropicais mais consumidos no Nordeste brasileiro é o caju (Anacardium occidentale L). A cultura do caju é uma das principais atividades nos estados do Ceará, Piauí e Rio Grande do Norte, tendo produção média de 1,5 milhões de kg por ano (SANTOS et al., 2007). O caju é firme, pequeno, em formato de pêra e é um fruto não climatério, sendo encontrado em três cores: amarelo, laranja e vermelho. Os frutos mais comumente comercializados são os amarelos e vermelhos (ASSUNÇÃO $\&$ MERCADANTE, 2003).
A produção brasileira comercializada no CEAGESP (Companhia de Entrepostos e Armazéns Gerais do Estado de São Paulo) foi de 2.108 toneladas no ano de 2005, segundo Agrianual (2007), sendo explorado somente 10\% do total da produção, na forma de fruto fresco ou produtos industriais como suco, polpa, doces e bebidas alcoólicas (EMBRAPA, 1999).

Até muito recentemente os pedúnculos eram vendidos em feiras locais, porém hoje alcançam supermercados em outras partes do país, localizadas a mais de $4000 \mathrm{~km}$ do local de produção (MORAIS et al., 2002). A ausência e/ou deficiência de técnicas adequadas de

\footnotetext{
'Engenheira Química, Doutora - Escola de Agronomia e Engenharia de Alimentos/EAEA - Universidade Federal de Goiás/UFG - Campus Samambaia Rodovia Goiânia/Nova Veneza, Km 0 - Cx. P. 131 - 74001-970 - Goiânia, GO - drilavras@yahoo.com.br

${ }^{2}$ Nutricionista, Doutora - Agroindústria, Alimentos e Nutrição/LAN - Universidade de São Paulo/USP - Escola Superior de Agricultura "Luiz de Queiroz"/ ESALQ - Avenida Pádua Dias, 11 - Agronomia - Cx. P. 9 -13418-900 - Piracicaba, SP - sgcbraza@esalq.usp.br

${ }^{3}$ Biólogo, Doutor - Laboratório de Irradiação de Alimentos e Radioentomologia/LIARE - Universidade de São Paulo/USP - Centro de Energia Nuclear na Agricultura/CENA - Avenida Centenário, 303 - Agronomia - 13400-970 - Piracicaba, SP - arthur@cena.usp.br

${ }^{4}$ Graduado em Ciência de Alimentos - Desenvolvimento de Produtos - Pastifício Selmi - Unidade Sumaré - Rodovia Virgínia Viel Campo Dall'orto, s/n, Km 1,9 - São Francisco - 13175-586 - Sumaré, SP - andersonoliveira@selmi.com.br

${ }^{5}$ Engenheira Agrônoma, Doutora - Agroindústria, Alimentos e Nutrição/LAN - Universidade de São Paulo/USP - Escola Superior de Agricultura "Luiz de Queiroz"/ESALQ - Avenida Pádua Dias, 11-Agronomia - Cx. P. 9-13418-900 - Piracicaba, SP - mhfspoto@esalq.usp.br

${ }^{6}$ Engenheiro Agrônomo, Doutor - Laboratório de Irradiação de Alimentos e Radioentomologia/LIARE - Universidade de São Paulo/USP - Centro de Energia Nuclear na Agricultura/CENA - Avenida Centenário, 303 - Agronomia - 13400-970 - Piracicaba, SP - jmwalder@cena.usp.br
} 
manuseio, transporte e armazenamento, associada à alta perecibilidade, geram perdas na produção de frutos. Essas perdas podem ser reduzidas pela utilização de processamento mínimo e outros métodos de conservação combinados (CHITARRA \& PRADO, 2002). Associada aos procedimentos pós-colheita normalmente empregados, a radiação gama, em baixos níveis de dose, tem mostrado ser um excelente método para prolongar a vida comercial das frutas, retardando os processos de amadurecimento e senescência, bem como reduzindo significativamente o apodrecimento causado por fungos e bactérias patogênicas (GERMANO et al., 1996).

O emprego da irradiação na pós-colheita de frutos tem como principal interesse a redução nos danos causados por doenças. Contudo, é também utilizada como método de conservação, prolongando o armazenamento pela diminuição na velocidade do amadurecimento e do brotamento de alguns produtos. Entretanto, apresenta alguns inconvenientes, pois dependendo da dosagem de irradiação, pode provocar escurecimento, amaciamento, aparecimento de depressões superficiais, amadurecimento anormal e perda de aroma e sabor nos produtos. Em pedúnculos de caju foram realizados estudos bioquímicos indicando que alterações no armazenamento são prejudiciais ao fruto, porém são necessários tais estudos para prolongamento da vida de prateleira do mesmo (NAGARAJA, 2007).

A aplicação de radiação ionizante pode alterar os componentes estruturais de alguns frutos dando-lhes um aspecto mais benéfico, aumentando sua firmeza. Baixas doses de irradiação resultam na hidrólise de alguns componentes resultando em maior vida útil do produto e conversão de amidos em açúcares. É comum encontrar frutas que foram irradiadas mais doces em relação às não irradiadas. A radiação gama em baixas doses tem sido utilizada para retardar o processo de amadurecimento e senescência, prolongando a vida pós-colheita de frutos. Níveis ótimos de radiação retardam a ocorrência de picos de respiração, porém as doses ótimas e máximas que o fruto pode tolerar dependem dos cultivares (THOMAS, 1986). Como vantagem adicional, a radiação ionizante induz pequenas alterações fisiológicas, fazendo com que haja diminuição ou mesmo paralização dos processos de maturação, prolongando sua vida de prateleira, incluindo aqui consideráveis vantagens econômicas (GERMANO et al., 1996).

Tendo em vista a manutenção da qualidade em pseudofrutos de caju, objetivou-se, neste trabalho, avaliar a influência da irradiação (diferentes doses) na qualidade e vida de prateleira do caju.

\section{MATERIALE MÉTODOS}

\section{Matéria-prima}

Os pedúnculos de caju vermelho maduro, proveniente de cajueiro anão precoce, utilizados no presente estudo foram obtidos no comércio de Piracicaba (SP). Foram obtidas 3 bandejas com 4 frutos cada para cada tratamento com três repetições. Os pedúnculos foram higienizados e embalados em bandejas de polietileno cobertas com filme plástico, irradiados num irradiador tipo Gammacell, com fonte de cobalto $\left({ }^{60} \mathrm{Co}\right)$, no Centro de Energia Nuclear na Agricultura (CENA/USP). As doses de irradiação empregadas foram de 0, 0,5 e 1,0 kGy. Após a irradiação os pedúnculos foram mantidos sob refrigeração $\left(4{ }^{\circ} \mathrm{C}\right)$. Foram retiradas 3 bandejas para cada tempo e para cada dose utilizada para a realização das análises.

Foram realizadas, em triplicata, as seguintes análises: sólidos solúveis totais (SST), $\mathrm{pH}$, vitamina $\mathrm{C}$, açúcares redutores, firmeza e cor. As análises foram realizadas aos 0, 3, 6, 9 dias de armazenamento.

As análises de cor e firmeza foram realizadas no CENA, e as demais no Laboratório de Nutrição Humana e Alimentos, da Escola Superior de Agricultura "Luiz de Queiroz".

\section{Métodos de análise}

\section{Açúcares redutores}

O método utilizado para obtenção dos teores de açúcares redutores foi segundo Miller (1959), no qual foram pesados $25 \mathrm{~g}$ de amostra e neutralizados a pH menor que 7 . Foi adicionado solução de ferrocianeto de potássio e acetato de zinco e em seguida filtrado. Foram acrescentados $50 \mathrm{~mL}$ de solução Fehling e levado para aquecimento. Filtrou-se a vácuo em cadinho (previamente tarado) e levouse à estufa a $105{ }^{\circ} \mathrm{C}$ por 1 hora. $\mathrm{O}$ cadinho foi pesado para obtenção do valor de óxido cuproso retido. De acordo com os valores obtidos de óxido cuproso, foram calculados os teores de açúcares redutores.

\section{Sólidos solúveis totais (SST)}

A medida de graus Brix foi obtida utilizando refratômetro Auto Abbe, conforme metodologia da AOAC (1992).

\section{Firmeza}

A firmeza dos pedúnculos foi obtida através de penetrômetro de mão, com haste cilíndrica de $30 \mathrm{~mm}$ de diâmetro, com ponta cônica (Force Dial - Wagner Instruments, modelo FDN 2) e as medidas foram tomadas na região central dos frutos, segundo Andrade et al. (2006). 


\section{Vitamina C}

A análise de ácido ascórbico (vitamina C) foi realizada segundo método proposto pela AOAC (1984) modificado por Benassi (1990). Para se evitar a perda de vitamina $\mathrm{C}$, a extração das amostras foi feita com solução de ácido oxálico $0,4 \%$.

\section{Potencial hidrogeniônico - pH}

Os valores de $\mathrm{pH}$ foram medidos em $\mathrm{pHmetro}$ calibrado (marca Tecnal), de acordo com metodologia descrita por AOAC (1992).

\section{Cor}

As medidas de cor foram realizadas com colorímetro Minolta CR-200 b com iluminante D65 e 8 mm de diâmetro de olho, previamente calibrado em superfície branca, de acordo com padrões preestabelecidos, segundo Andrade et al. (2006). Foram obtidos os valores de $L, a$ e $b$, tomando a leitura em 3 cubos por repetição de cada tratamento. Os valores de $C$ (croma) e $H$ (ângulo Hue, que mede a tonalidade) foram calculados posteriormente através das seguintes fórmulas:

$$
C=\left(a^{2}+b^{2}\right)^{0,5} \quad \text { e } \quad H^{0}=\operatorname{arctg} \frac{b}{a}
$$

\section{Análise Estatística}

O delineamento experimental foi realizado ao acaso, em esquema fatorial $3 \times 4$ (doses de irradiação e tempos de armazenamento). Foram realizadas 3 repetições, sendo que cada amostra experimental consistiu de uma bandeja com 4 cajus. Os dados foram submetidos à análise de variância e as médias comparadas pelo teste de Tukey ( $\mathrm{pd} \leq 0,01$ ), com utilização do programa estatístico SAS Institute (1998).

\section{RESULTADOS E DISCUSSÃO}

O teor de açúcares usualmente aumenta com o amadurecimento dos frutos por processos de biossíntese ou pela degradação de polissacarídeos. No dia inicial, logo após a irradiação os teores de açúcares redutores aumentaram em função das doses de irradiação utilizadas (Tabela 1). Após o armazenamento, todos os tratamentos resultaram em aumento do teor de açúcares redutores devido ao processo de maturação, sendo que somente a dose de $1 \mathrm{kGy}$ diferiu dos demais tratamentos.

As doses de irradiação e o tempo de armazenamento utilizados influenciaram nos valores de açúcares redutores dos frutos, já que baixas doses de irradiação podem converter amido em açúcar (KOVACS et al., 1995), e que a hidrólise do amido e conseqüente acúmulo de açúcares, é uma mudança fundamental para as características organolépticas do fruto (CHITARRA \& CHITARRA, 2006), o que explicaria os teores mais altos serem apresentadas na dose de $0,5 \mathrm{kGy}$.

Os sólidos solúveis dos pedúnculos de caju, foram mais influenciados pela dose de 1,0 kGy, na qual foram encontrados os maiores valores no tempo inicial. Esse comportamento se manteve com o armazenamento, ocorrendo diminuição dos valores de graus Brix, independente das doses de irradiação utilizadas como pode ser observado na Tabela 2.

Tabela 1 - Teores de açúcares redutores $(\mathrm{mg} / \mathrm{g})$ em pseudofrutos de caju irradiados e armazenados a $4{ }^{\circ} \mathrm{C}$.

\begin{tabular}{ccccc}
\hline & \multicolumn{4}{c}{ Açúcares Redutores/Tempo de armazenamento à $4{ }^{\circ} \mathrm{C}$ (dias) } \\
\hline Dose $(\mathrm{kGy})$ & 0 dia & 3 dias & 6 dias & 9 dias \\
$0($ Controle $)$ & $5,82^{\mathrm{Aa}} \pm 0,01$ & $5,63^{\mathrm{Aa}} \pm 0,11$ & $8,28^{\mathrm{Ab}} \pm 0,48$ & $10,58^{\mathrm{Ac}} \pm 0,31$ \\
0,5 & $10,24^{\mathrm{Ba}} \pm 0,01$ & $7,24^{\mathrm{Bb}} \pm 0,16$ & $10,25^{\mathrm{Ba}} \pm 0,24$ & $14,20^{\mathrm{Bc}} \pm 0,17$ \\
1,0 & $9,91^{\mathrm{Ca}} \pm 0,01$ & $5,61^{\mathrm{Ab}} \pm 0,21$ & $6,18^{\mathrm{Cb}} \pm 0,21$ & $11,48^{\mathrm{Ac}} \pm 0,42$ \\
\hline
\end{tabular}

Letras maiúsculas diferentes na vertical e letras minúsculas diferentes na horizontal indicam diferença significativa a $1 \%$ entre doses e tempos, respectivamente, pelo teste de Tukey.

Tabela 2 - Valores de sólidos solúveis totais ( ${ }^{\circ}$ Brix) em pseudofrutos de caju irradiados e armazenados a $4{ }^{\circ} \mathrm{C}$.

\begin{tabular}{ccccc}
\hline & \multicolumn{4}{c}{ Sólidos Solúveis totais /Tempo de armazenamento à $4^{\circ} \mathrm{C}$ (dias) } \\
\hline Dose $(k G y)$ & 0 & 3 & 6 & 9 \\
$0($ Controle $)$ & $11,90^{\mathrm{Aa}} \pm 0,01$ & $9,00^{\mathrm{Ab}} \pm 0,01$ & $10,00^{\mathrm{Ac}} \pm 0,01$ & $8,80^{\mathrm{Ad}} \pm 0,01$ \\
0,5 & $11,90^{\mathrm{Aa}} \pm 0,01$ & $11,00^{\mathrm{Bb}} \pm 0,01$ & $11,40^{\mathrm{Bc}} \pm 0,01$ & $8,80^{\mathrm{Ad}} \pm 0,01$ \\
1,0 & $12,20^{\mathrm{Ba}} \pm 0,01$ & $12,10^{\mathrm{Cb}} \pm 0,01$ & $10,20^{\mathrm{Cc}} \pm 0,01$ & $9,20^{\mathrm{Bd}} \pm 0,01$ \\
\hline
\end{tabular}

Letras maiúsculas diferentes na vertical e letras minúsculas diferentes na horizontal indicam diferença significativa a $1 \%$ entre doses e tempos, respectivamente, pelo teste de Tukey. 
A irradiação influenciou a firmeza do caju, diminuindo os valores em função do aumento das doses, na primeira avaliação realizada. Os valores aumentaram com o passar do tempo de armazenamento (Tabela 3), porém para o controle e 1 kGy não houve variação significativa.

A aplicação de radiação ionizante pode alterar os componentes estruturais de alguns frutos dando-lhes um aspecto mais firme. Thomas (1986) estudando amadurecimento de bananas irradiadas, notou diminuição da taxa de amolecimento, e que após 30 dias de armazenamento, os frutos irradiados com as doses mais altas se mostraram mais firmes que as frutas irradiadas com a doses menores. A diminuição da firmeza dos frutos encontrada quando se compara as doses de irradiação, pode ser explicada segundo Chitarra \& Chitarra (1990), pela degradação do amido e conversão de açúcares, degradação da parede celular e a solubilização de substâncias pécticas.

Os teores encontrados de vitamina $\mathrm{C}$, no tempo inicial para os pseudofrutos de caju, foram diminuindo com o emprego de doses maiores de irradiação (Tabela 4). A dose que mais influenciou os teores de ácido ascórbico foi a de 1,0 kGy, que apresentou os valores mais baixos.

Após 9 dias de armazenamento a diferença entre $o$ controle e a dose de $0,5 \mathrm{kGy}$ não existia mais, somente o tratamento de $1 \mathrm{kGy}$ diferiu dos demais. Durante o armazenamento, o teor de vitamina $\mathrm{C}$ decresceu $42,7 \%$ do teor inicialmente presente nos frutos comparando todas as doses empregadas e o tempo de armazenamento. Os resultados indicam que a irradiação influencia nos teores de vitamina $\mathrm{C}$ de acordo com a dose empregada, e que a quantidade perdida durante o processo pode ser comparada aos demais processos empregados, como conservação por refrigeração, para a extensão de vida de prateleira dos produtos.

Apesar do decréscimo na concentração de ácido ascórbico ao final de 9 dias de armazenamento, os valores encontrados estão abaixo dos valores relatados por Damasceno Júnior \& Bezerra (2002) que estudaram pedúnculos de caju. Morais et al. (2002) constataram perdas de 6,5\% de ácido ascórbico em pedúnculos de caju sob refrigeração, durante 25 dias em atmosfera modificada.

Para Assunção \& Mercadante (2003) os teores de vitamina $\mathrm{C}$ encontrados para pedúnculos de caju de várias variedades foram de 104 a $118 \mathrm{mg} / 100 \mathrm{~g}$ polpa sem armazenamento. Esses resultados estão abaixo dos encontrados no período inicial (Tabela 4). Essa diferença pode ser atribuída ao local de colheita dos frutos e o processo de conservação empregado.

Os valores de $\mathrm{pH}$ aumentaram (Tabela 5) com o aumento das doses de irradiação, com o armazenamento a $4^{\circ} \mathrm{C}$ diminuíram para os pseudofrutos que sofreram irradiação e aumentam para o controle. Essa alteração pode ter ocorrido devido a temperatura de armazenamento em conjunto com o efeito da irradiação, que pode ter provocado desnaturação de membranas. Em conseqüência disso, o $\mathrm{pH}$ mais baixo do conteúdo do vacúolo em contato com o pH externo pode sofrer aumento (MURATA, 1990). $\mathrm{O}$ aumento de $\mathrm{pH}$ pode então provocar alteração na cor do pedúnculo do caju, já que as antocianinas são vermelhas em pH baixo (pH 3,0), incolores em $\mathrm{pH}$ de 4 a 5 e azulada em

Tabela 3 - Resistência à penetração (firmeza em $\mathrm{N}$ ) de pseudofrutos de caju irradiados e armazenados à $4{ }^{\circ} \mathrm{C}$.

\begin{tabular}{ccccc}
\hline & \multicolumn{4}{c}{ Resistência a penetração/Tempo de armazenamento à $4{ }^{\circ} \mathrm{C}$ (dias) } \\
\hline Dose $(\mathrm{kGy})$ & 0 & 3 & 6 & 9 \\
$0($ Controle $)$ & $0,82^{\mathrm{Aa}} \pm 0,16$ & $1,11^{\mathrm{Ba}} \pm 0,11$ & $1,13^{\mathrm{Ba}} \pm 0,13$ & $1,23^{\mathrm{Aa}} \pm 0,15$ \\
0,5 & $0,34^{\mathrm{Ba}} \pm 0,08$ & $0,48^{\mathrm{Aab}} \pm 0,05$ & $0,72^{\mathrm{Ab}} \pm 0,06$ & $1,13^{\mathrm{Ac}} \pm 0,13$ \\
1,0 & $0,31^{\mathrm{Ba}} \pm 0,03$ & $0,59^{\mathrm{Aa}} \pm 0,31$ & $0,69^{\mathrm{Aa}} \pm 0,19$ & $0,79^{\mathrm{Ba}} \pm 0,16$ \\
\hline
\end{tabular}

Letras maiúsculas diferentes na vertical e letras minúsculas diferentes na horizontal indicam diferença significativa a $1 \%$ entre doses e tempos, respectivamente, pelo teste de Tukey.

Tabela 4 - Teores de vitamina C (mg/100 g) em pseudofrutos de caju irradiados e armazenados a $4{ }^{\circ} \mathrm{C}$.

\begin{tabular}{ccccc}
\hline \multicolumn{5}{c}{ Teor de vitamina C / Tempo de armazenamento a $4^{\circ} \mathrm{C}$ (dias) } \\
\hline Dose $(\mathrm{kGy})$ & 0 & 3 & 6 & 9 \\
$0($ Controle $)$ & $201,11^{\mathrm{Aa}} \pm 0,96$ & $170,80^{\mathrm{Ab}} \pm 1,03$ & $109,38^{\mathrm{Ac}} \pm 1,57$ & $98,96^{\mathrm{Ad}} \pm 2,38$ \\
0,5 & $143,34^{\mathrm{Ba}} \pm 2,89$ & $105,20^{\mathrm{Bb}} \pm 1,02$ & $96,36^{\mathrm{Bc}} \pm 2,39$ & $93,23^{\mathrm{Ac}} \pm 0,90$ \\
1,0 & $110,56^{\mathrm{Cb}} \pm 0,96$ & $148,94^{\mathrm{Ca}} \pm 1,78$ & $85,94^{\mathrm{Bc}} \pm 1,56$ & $63,54^{\mathrm{Bd}} \pm 0,90$ \\
\hline
\end{tabular}

Letras maiúsculas diferentes na vertical e letras minúsculas diferentes na horizontal indicam diferença significativa a $1 \%$ entre doses e tempos, respectivamente, através do Teste de Tukey. 
pH mais elevado (MAZZA \& BROUILLARD, 1987). Segundo Figueiredo et al. (2007) ocorreu desestabilização da cor vermelha de pedúnculo de caju ao longo do armazenamento, o que pode explicar a alteração dos valores de C (Tabela 7).

Com outras frutas, como a pitanga, armazenadas refrigeradas ou congeladas também observaram alteração significativa no valor de pH dos frutos (MÉLO et al., 2000). A dose de radiação também apresentaram efeito significativo sobre o $\mathrm{pH}$ de suco de laranja e também em uva tipo "Itália", provocando aumento do mesmo (DOMARCO et al., 1999; IEMMA et al., 1999).

Com relação a característica da cor, os valores de $\mathrm{L}$ para os pseudofrutos de caju variaram entre o controle e a dose de $0,5 \mathrm{kGy}$, não só no dia inicial, mas também durante o armazenamento a $4^{\circ} \mathrm{C}$, por 9 dias (Tabela 6).

Os valores de $C$ (Tabela 7) indicam que o método de irradiação inibiu o desenvolvimento da coloração avermelhada dos frutos o que está diretamente associado à maturação pelos consumidores. Quanto ao tempo de armazenamento, os menores valores foram observados para o terceiro dia de armazenamento.

Os valores de $H$ (Tabela 8) diferenciaram-se estatisticamente entre a dose de $1 \mathrm{kGy}$ e demais tratamentos. Os tempos, mostrando a dose de $0,5 \mathrm{kGy}$ ser mais influente nos primeiros dias, e a dose de $1 \mathrm{kGy}$ no nono dia, indicando que a tonalidade foi alterada durante o período de armazenamento a $4^{\circ} \mathrm{C}$ por 9 dias.

Tabela 5 - Valores de pH em pseudofrutos de caju irradiados e armazenados a $4{ }^{\circ} \mathrm{C}$.

\begin{tabular}{ccccc}
\hline \multicolumn{5}{c}{$\mathrm{pH} /$ Tempo de armazenamento a $4^{\circ} \mathrm{C}$ (dias) } \\
\hline Dose (kGy) & 0 & 3 & 6 & 9 \\
0 (Controle) & $4,61^{\mathrm{Aa}} \pm 0,01$ & $4,61^{\mathrm{Aa}} \pm 0,01$ & $4,72^{\mathrm{Ab}} \pm 0,01$ & $4,70^{\mathrm{Ab}} \pm 0,01$ \\
0,5 & $5,01^{\mathrm{Ba}} \pm 0,01$ & $5,14^{\mathrm{Bb}} \pm 0,11$ & $4,75^{\mathrm{Bc}} \pm 0,01$ & $4,59^{\mathrm{Bd}} \pm 0,01$ \\
1,0 & $5,05^{\mathrm{Ca}} \pm 0,01$ & $4,68^{\mathrm{Ab}} \pm 0,01$ & $4,69^{\mathrm{Cb}} \pm 0,01$ & $4,49^{\mathrm{Cc}} \pm 0,01$ \\
\hline
\end{tabular}

Letras maiúsculas diferentes na vertical e letras minúsculas diferentes na horizontal indicam diferença significativa a $1 \%$ entre doses e tempos, respectivamente, pelo teste de Tukey.

Tabela 6 - Valores de $L$ para o armazenamento de pseudofrutos de caju irradiados e armazenados à $4{ }^{\circ} \mathrm{C}$.

\begin{tabular}{ccccc}
\hline & \multicolumn{4}{c}{ Valores de L/Tempo de armazenamento à $4^{\circ} \mathrm{C}$ (dias) } \\
\hline Dose $(\mathrm{kGy})$ & 0 & 3 & 6 & 9 \\
(0) Controle & $53,43^{\mathrm{Bb}} \pm 2,84$ & $57,33^{\mathrm{Aba}} \pm 3,89$ & $61,97^{\mathrm{Aa}} \pm 6,69$ & $55,73^{\mathrm{Bb}} \pm 5,16$ \\
0,5 & $60,73^{\mathrm{Aa}} \pm 3,64$ & $61,33^{\mathrm{Aa}} \pm 2,14$ & $53,27^{\mathrm{Bb}} \pm 5,15$ & $57,87^{\mathrm{Aa}} \pm 9,19$ \\
1,0 & $56,87^{\mathrm{Bab}} \pm 6,64$ & $56,63^{\mathrm{Ba}} \pm 3,47$ & $63,93^{\mathrm{Aa}} \pm 7,67$ & $58,73^{\mathrm{Ba}} \pm 6,31$ \\
\hline
\end{tabular}

Letras maiúsculas diferentes na vertical e letras minúsculas diferentes na horizontal indicam diferença significativa a $1 \%$ entre doses e tempos, respectivamente, pelo teste de Tukey.

Tabela 7 - Valores de $C$ para o armazenamento de pseudofrutos de caju irradiados e armazenados à $4{ }^{\circ} \mathrm{C}$.

\begin{tabular}{ccccc}
\hline \multicolumn{4}{c}{ Valores de C/Tempo de armazenamento à $4^{\circ} \mathrm{C}$ (dias) } \\
\hline Dose $(\mathrm{kGy})$ & 0 & 3 & 6 & 9 \\
(0) Controle & $51,11^{\mathrm{Aa}} \pm 3,44$ & $40,71^{\mathrm{Cc}} \pm 5,23$ & $46,91^{\mathrm{Ab}} \pm 2,25$ & $47,09^{\mathrm{Ab}} \pm 2,22$ \\
0,5 & $47,80^{\mathrm{ABa}} \pm 3,97$ & $47,84^{\mathrm{Aa}} \pm 1,77$ & $46,86^{\mathrm{Aa}} \pm 3,07$ & $47,14^{\mathrm{Aa}} \pm 1,51$ \\
1,0 & $47,59^{\mathrm{Bab}} \pm 4,79$ & $43,97^{\mathrm{Bc}} \pm 3,64$ & $50,93^{\mathrm{Aa}} \pm 6,32$ & $44,83^{\mathrm{Bab}} \pm 5,04$ \\
\hline
\end{tabular}

Letras maiúsculas diferentes na vertical e letras minúsculas diferentes na horizontal indicam diferença significativa a $1 \%$ entre doses e tempos, respectivamente, pelo Teste de Tukey.

Tabela 8 -Valores de $H$ para o armazenamento de pseudofrutos de caju irradiados e armazenados à $4{ }^{\circ} \mathrm{C}$.

\begin{tabular}{ccccc}
\hline & \multicolumn{4}{c}{ Valores de H /Tempo de armazenamento à $4{ }^{\circ} \mathrm{C}$ (dias) } \\
\hline Dose (kGy) & 0 & 3 & 6 & 9 \\
0 (Controle) & $57,04^{\mathrm{Bb}} \pm 2,84$ & $52,88^{\mathrm{Bc}} \pm 3,89$ & $51,69^{\mathrm{Cc}} \pm 6,69$ & $67,94^{\mathrm{Aa}} \pm 5,16$ \\
0,5 & $59,04^{\mathrm{Bc}} \pm 2,61$ & $62,25^{\mathrm{Ab}} \pm 2,14$ & $73,64^{\mathrm{Aa}} \pm 5,15$ & $53,21^{\mathrm{Cd}} \pm 4,39$ \\
1,0 & $74,95^{\mathrm{Aa}} \pm 6,54$ & $54,99^{\mathrm{Bd}} \pm 3,47$ & $68,67^{\mathrm{Bb}} \pm 7,26$ & $61,86^{\mathrm{Bc}} \pm 3,31$ \\
\hline
\end{tabular}

Letras maiúsculas diferentes na vertical e letras minúsculas diferentes na horizontal indicam diferença significativa a $1 \%$ entre doses e tempos, respectivamente, pelo Teste de Tukey. 


\section{CONCLUSÃO}

A irradiação é um bom método para conservação e extensão da vida útil do fruto. A qualidade dos frutos foi influenciada pelo processo, tornando os frutos mais macios. Quanto maior as doses além de contribuir no aspecto de palatabilidade dos frutos, a variação nos valores de sólidos solúveis indicam a quantidade de açúcar presente na polpa.

\section{REFERÊNCIAS BIBLIOGRÁFICAS}

AGRIANUAL. Anuário da agricultura brasileira. São Paulo: FNP Consultoria \& Comércio, 2007.

ANDRADE, S. R. R.; ARRUDA, M. C.; JACOMINO, A. P.; SPOTO, M. H. F. Atividade respiratória e produção de etileno de mamões minimamente processados tratados com cloreto de cálcio e ácido ascórbico. In: ENCONTRO NACIONAL SOBRE PROCESSAMENTO MÍNIMO DE FRUTAS E HORTALIÇAS, 4.; SIMPÓSIO IBEROAMERICANO DE VEGETAIS FRESCOS CORTADOS, 2006, São Paulo, SP. Anais... São Paulo, 2006. v. 4, p. 143.

\section{ASSOCIATION OF OFFICIAL ANALYTICAL} CHEMISTS. Official methods of the Association of Official the Analytical Chemists. 12. ed. Arlington, 1984. $1141 \mathrm{p}$.

\section{ASSOCIATION OF OFFICIAL ANALYTICAL CHEMISTS. Official methods of the Association of Official the Analytical Chemists. 14. ed. Washington, DC, 1992. v. 2.}

ASSUNÇÃO, R. B.; MERCADANTE, A. Z. Carotenoids and ascorbic acids from cashew apple (Anacardium ocidentale L.): variety and geographic effects. Food Chemistry, New York, v. 81, n. 4, p. 495-502, June 2003.

BENASSI, M. T. Análise do efeito de diferentes parâmetros na estabilidade de vitamina $C \mathrm{em}$ vegetais processados. 1990. 159 f. Dissertação (Mestrado) Universidade de Estadual de Campinas, Campinas, 1990.

CHITARRA, A. B.; PRADO, M. E. T. Tecnologia de armazenamento pós-colheita para frutos e hortaliças in natura. Lavras: UFLA/FAEPE, 2002. 112 p.

ChITARRA, M. I. F.; CHITARRA, A. B. Pós-colheita de frutos e hortaliças: fisiologia e manuseio. Lavras: UFLA/FAEPE, 1990. 293 p.
ChITARRA, M. I. F.; CHITARRA, A. B. Pós-colheita de frutas e hortaliças. Lavras: UFLA/FAEPE, 2006. 256 p.

DAMASCENO JUNIOR, J. A.; BEZERRA, F. C. Qualidade de pedúnculo de cajueiro-anão precoce cultivado sob irrigação e submetido a diferentes sistemas de condução e espaçamento. Revista Brasileira de Fruticultura, Jaboticabal, v. 24, n. 1, 2002. Disponível em: <http:///www. scielo.bri

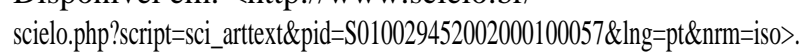
Acesso em: 14 maio 2007.

DOMARCO, R. E.; SPOTO, M. H. F.; BLUMER, L.; WALDER, J. M. M. Sineergia da radiação ionizante e do aquecimento na vida de prateleira da uva "Itália".

Scientia Agricola, Piracicaba, v. 56, n. 4, p. 982-986, 1999.

EMPRESA BRASILEIRA DE PESQUISA

AGROPECUÁRIA. Banco ativo de germoplasma de caju: variabilidade, caracterização e utilização 1999. Disponível em: <ihttp://ceinfo.cnpatembrapa.bry arquivos/artigo $2584 . p \mathrm{df}$. Acesso em: 16 abr. 2006.

FIGUEIREDO, R. W.; LAJOLO, F. M.; ALVES, R. E.; FIGUEIRAS, H. A. C.; MAIA, G. A.; SOUSA, P. H. M. Qualidade de pedúnculos de caju submetidos à aplicação pós-colheita de cálcio e armazenadas sob refrigeração. Pesquisa Agropecuária Brasileira, Brasília, v. 42, n. 4, p. 475-482, 2007.

GERMANO, R. M. de A.; ARTHUR, V.; WIENDL, F. M. Conservação pós-colheita de abacates Persia americana Mill., variedades Fortuna e Quintal, por irradiação.

Scientia Agricola, Piracicaba, v. 53, 1996. Disponível em: Shttp://www.scielo.br/

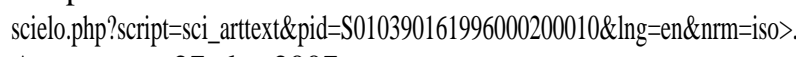
Acesso em: 27 abr. 2007.

IEMMA, J.; ALCARDE, A. R.; DOMARCO, R. E.; SPOTO, M. H. F.; BLUMER, L.; MATRAIA, C. Radiação gama na conservação do suco natural de laranja. Scientia Agricola, Piracicaba, v. 56, n. 4, p. 1193-1198, 1999. Suplemento.

KOVACS, E.; BALLA, G.; NESSINGER, A. The effect of irradiation on sweet cherries. Acta Alimentaria, v. 24, p. 331-343, 1995.

MAZZA, G.; BROUILLARD, R. Recent developments in the stabilization of anthocyanins in food products. Food Chemistry, New York, v. 25, n. 3, p. 207-225, 1987. 
MÉLO, E. A.; LIMA, V. L. A. G.; NASCIMENTO, P. P. Temperatura no armazenamento de pitanga. Scientia Agricola, Piracicaba, v. 57, n. 4, p. 629-634, dez. 2000.

MILLER, G. L. Use of dinitrosalicylic acid reagent for determination of reducing sugars. Analytical Chemistry, v. 31, n. 3, p. 426-428, Mar. 1959.

MORAIS, A. S.; MAIA, G. A.; FIGUEIREDO, R. W.; ALVES, R. E.; FILGUEIRAS, H. A. C.; MOURA, C. F. H. Armazenamento refrigerado sob atmosfera modificada de pedúnculos de cajueiro-anão-precoce dos clones $\mathrm{CCP}$ 76, END-157, END-183 e END-189. Revista Brasileira de Fruticultura, Jaboticabal, v. 24, n. 3, p. 647-650, dez. 2002.

MURATA, T. Relation of chilling stress to membrane permeability. In: WANG, C. Y. Chilling injury of horticultural crops. Boca Raton: CRC, 1990. p. 201-209.
NAGAJATA, K. V. Biochemistry of cashew (Anacardium occidentale L): a review. Journal Food Science Technology, v. 44, n. 1, p. 1-9, Feb. 2007.

SANTOS, R. P.; SANTIAGO, A. A. X.; GADELHA, C. A. A.; CAJAZEIRAS, J. B.; CAVADE, B. S.; MARTINS, J. L.; OLIVEIRA, T. M.; BEZERRA, G. A.; SANTOS, R. P.; FREIRE, V. N. production and characterization of the cashew (Anacardium occidentale L.) penduncle bagasse ashes. Journal of Food Engineering, v. 79, n. 4, p. 1432-1437, Apr. 2007.

SAS INSTITUTE. SAS user's guide: statistic. Version 6. 12. ed. Cary, 1998. 846 p.

THOMAS, P. Radiation preservation of foods of plant origin: III, tropical fruits: bananas mangoes and papayas. Critical Review in Food Science and Nutrition, v. 23, n. 1, p. 147-205, Feb. 1986. 